\title{
CiBER-seq dissects genetic networks by quantitative CRISPRi profiling of expression phenotypes
}

\author{
Ryan Muller', Zuriah A. Meacham1, Lucas Ferguson', Nicholas T. Ingolia 1,2,"
}

\begin{abstract}
1Department of Molecular and Cell Biology and ${ }^{2}$ California Institute for Quantitative Biosciences, University of California, Berkeley, Berkeley, CA 94720, USA
\end{abstract}

${ }^{*}$ Correspondence: ingolia@berkeley.edu

To realize the promise of CRISPR/Cas9-based genetics, approaches are needed to quantify a specific, molecular phenotype across genome-wide libraries of genetic perturbations. We address this challenge by profiling transcriptional, translational, and post-translational reporters using CRISPR interference with barcoded expression reporter sequencing (CiBER-seq). Our barcoding approach connects an entire library of guides to their individual phenotypic consequences using pooled sequencing. We show that CiBER-seq profiling fully recapitulates the integrated stress response (ISR) pathway in yeast. Genetic perturbations causing uncharged tRNA accumulation activated ISR reporter transcription. Surprisingly, tRNA insufficiency also activated the reporter, independent of the Gcn2 kinase that senses uncharged tRNAs. By uncovering alternate triggers for ISR activation, we illustrate how precise, comprehensive CiBER-seq profiling provides a powerful and broadly applicable tool for dissecting genetic networks.

\section{INTRODUCTION}

CRISPR-Cas9 has emerged as a powerful and versatile tool for creating precise, programmable genetic perturbations (1). CRISPR-based knockout (2-4) and knockdown (5) approaches now enable systematic, genome-wide genetic analysis in a wide range of cells and organisms. The Cas9 protein binds short RNAs that guide this protein-RNA complex to complementary sites in the genome, where it can induce mutations or silence promoters (1). Libraries of guide RNAs, each targeting one individual gene, can be used to create a population of cells that each express one distinct guide (6-8). The phenotype of each cell then reflects the impact of the single guide that it expresses. It is straightforward to identify guides that affect cell survival or proliferation, but growth is a crude phenotype that is poorly suited to address many important biological questions (9). The scope of CRISPR-based genetics would be expanded by improved techniques to measure more specific and relevant phenotypes across this diverse population and link these measurements back to individual guides.
Molecular phenotypes, such as the expression level of a critical gene or the stability of a key protein, provide a focused and sensitive gauge for many aspects of cell physiology. We present an approach for profiling a transcriptional, translational, or post-translational regulatory response comprehensively across CRISPR-based perturbations genome-wide. We adapt barcoded expression reporters (10) to produce quantitative phenotypic profiles from bulk sequencing of highly diverse populations. These profiles also enable high-precision genetic interaction analyses, which use double mutant phenotypes to map the structure of regulatory networks (11). This direct sequencing approach offers significant advantages over fluorescent reporters for CRISPR-based genetics. Fluorescence phenotypes are typically analyzed by cell sorting (9), which imposes bottlenecks on the cell population size and discretizes quantitative fluorescence measurements into a few broad gates. Our approach circumvents both of these limitations. It also complements broader expression profiles from single-cell approaches such as Perturb-Seq $(12,13)$, CRISP-Seq (14), and CROP-Seq (15), which cannot capture enough 
cells to approach genome-scale coverage. These technically challenging studies each analyzed fewer than a hundred genes, and in fact, some relied on fluorescent reporter screens to choose a small, tractable set of guides for single-cell experiments (12). Better ways to profile molecular phenotypes across genome-scale guide libraries thus stand to benefit many areas of biology.

Here, we combine CRISPR interference with barcoded expression reporter sequencing (CiBER-seq) to measure cellular responses provoked by guide RNA-mediated knockdown. Using CiBER-seq, we analyze the integrated stress response (ISR) in budding yeast. We confirm the known pathway for ISR activation by amino acid starvation, which leads to the accumulation of uncharged tRNAs and inhibitory phosphorylation of the translation initiation factor eIF2 $\alpha$ (16). Surprisingly, we also find that $\mathrm{tRNA}$ depletion triggers the ISR through a distinct, uncharacterized mechanism that does not require eIF $2 \alpha$ phosphorylation. We demonstrate an indirect CiBER-seq approach to measure translational and post-translational regulation, and use this approach to show that ISR activation by $\mathrm{tRNA}$ depletion nonetheless results from translational control independent of eIF $2 \alpha$ phosphorylation. By analyzing doubleknockdown phenotypes in dual-guide CiBERseq, we uncover epistatic interactions that suggest a role for actin cytoskeletal components in mediating this non-canonical ISR. Our results illustrate the power of CiBER-Seq to provide new insights even in well-studied regulatory pathways and highlight the flexibility and broad applicability of our technique.

\section{RESULTS}

\section{Barcoded expression reporters link transcriptional responses with guide RNA- mediated perturbations in massively parallel screens.}

CiBER-Seq relies on massively parallel measurements of reporter expression in a diverse population by deep sequencing of short sequence "barcodes" embedded in the reporter transcript (10). The underlying abundance of
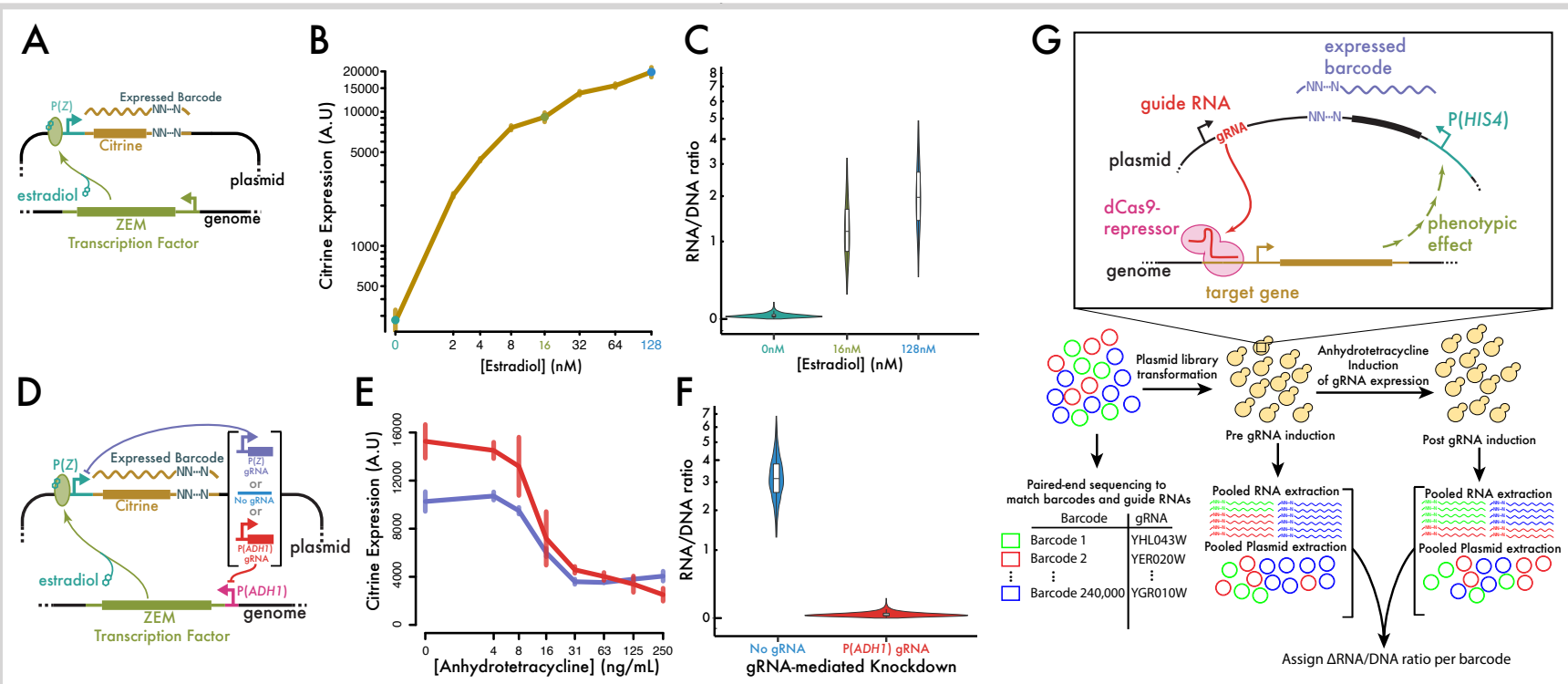

Fig. 1. Barcoded expression reporters accurately link guide RNAs with transcriptional phenotypes. (A) Schematic of synthetic ZEM transcription factor and $P(Z)$ promoter. (B, C) Expression measurements by (B) fluorescence and (C) RNA/DNA barcode count ratio show quantitative agreement. Inducer conditions for barcode sequencing in (C) are color-coded and marked with dots on the graph in (B). (D) Schematic of guide RNA effects on the synthetic transcription factor / promoter pair. (E) Fluorescent output is negatively correlated with guide RNA induction level. (F) Barcode sequencing expression measurements reflect effects of knockdown. (G) Schematic of CiBER-Seq profiling experiment. 
cells with each barcode can vary in this population, however, and so we set out to measure the transcriptional output from each barcode by normalizing the RNA abundance of that barcode against its DNA abundance. We first validated the use of barcode sequencing to measure expression using an artificial inducible promoter, $P(Z)(17)$, to drive the expression of a barcoded fluorescent reporter, allowing us to vary transcription of the reporter (fig. S1A) and compare fluorescence levels with barcode sequencing results. We generated three highdiversity barcoded libraries, each incorporating a distinct library sequence tag alongside random nucleotide barcodes, and treated these tagged populations with different levels of inducer (Fig. 1A). We measured fluorescence levels in each of these populations (Fig. 1B), and pooled them together to test our ability to measure expression differences in complex populations. The abundance of individual barcodes varied widely in each population (fig. S1D and S1E), but the RNA-to-DNA ratio showed a much tighter distribution that matched quantitatively the fluorescence levels (Fig. 1C).

Having established our ability to track transcriptional output through barcode sequencing, we next sought to link distinct barcoded reporters with guide RNAs that perturb reporter expression (Fig. 1D). The inducible promoter driving our reporter relies on an exogenous, chimeric transcription factor, ZEM (17). CRISPRi-mediated knockdown of this transcription factor, or the inducible $\mathrm{P}(\mathrm{Z})$ promoter itself, both reduced reporter fluorescence dramatically (Fig. 1E and fig. S1B and $\mathrm{C}$ ). We then carried out pooled sequencing on two high-complexity barcode libraries, one linked to the guide RNA that targeted the exogenous transcription factor. Barcode sequencing revealed a 91-fold reduction in reporter expression caused by transcription factor knockdown (Fig. 1F), demonstrating our ability to measure CRISPRi effects through a linked, barcoded reporter.
Based on these results, we devised a strategy for genome-wide CRISPR interference with barcode expression reporter sequencing (CiBER-seq). We linked each guide sequence in a comprehensive yeast guide RNA library with random nucleotide barcodes and determined the linkage between barcodes and guides by paired-end sequencing (Fig. 1G) (18). Our library contains 10 guides per gene ( 60,000 in total) and $\sim 240,000$ distinct barcodes ( $\sim 4$ per guide on average). By linking multiple barcodes with each guide, we were able to obtain independent measurements of guide effects within a single experiment. To control for barcode-specific effects on RNA stability, we drove guide RNA expression from a tetracyclineinducible promoter (19) and compared barcode expression before and after guide induction. Inducible CRISPRi also facilitates measurements of guides with strong fitness effects by allowing us to propagate cells without guide induction and thereby avoid the premature loss of guides with growth defects. Finally, we noted that barcode DNA-seq showed substantially more noise than barcode RNA-seq (fig. S1D and S1E), which we attribute to PCR amplification. In order to reduce this noise and improve the precision of our expression measurements, we constructed subsequent DNA libraries using linear pre-amplification by in vitro transcription (18).

\section{CiBER-seq recapitulates known genetic regulators of integrated stress response and identifies new regulators related to $T R N A$ insufficiency}

We first applied CiBER-Seq to survey genetic perturbations that activated a transcriptional reporter for the yeast integrated stress response (ISR), also known as the general amino acid control (GAAC) response. This deeply conserved pathway upregulates biosynthetic genes in response to elevated levels of uncharged tRNAs that arise during amino acid starvation (16). We constructed a guide library with barcodes driven by the promoter $P(H I S 4)$, which is activated by the ISR transcription factor Gcn 4 when 
bioRxiv preprint doi: https://doi.org/10.1101/2020.03.29.015057; this version posted March 31, 2020. The copyright holder for this preprint (which was not certified by peer review) is the author/funder, who has granted bioRxiv a license to display the preprint in perpetuity. It is made available under aCC-BY 4.0 International license.

Muller et al.
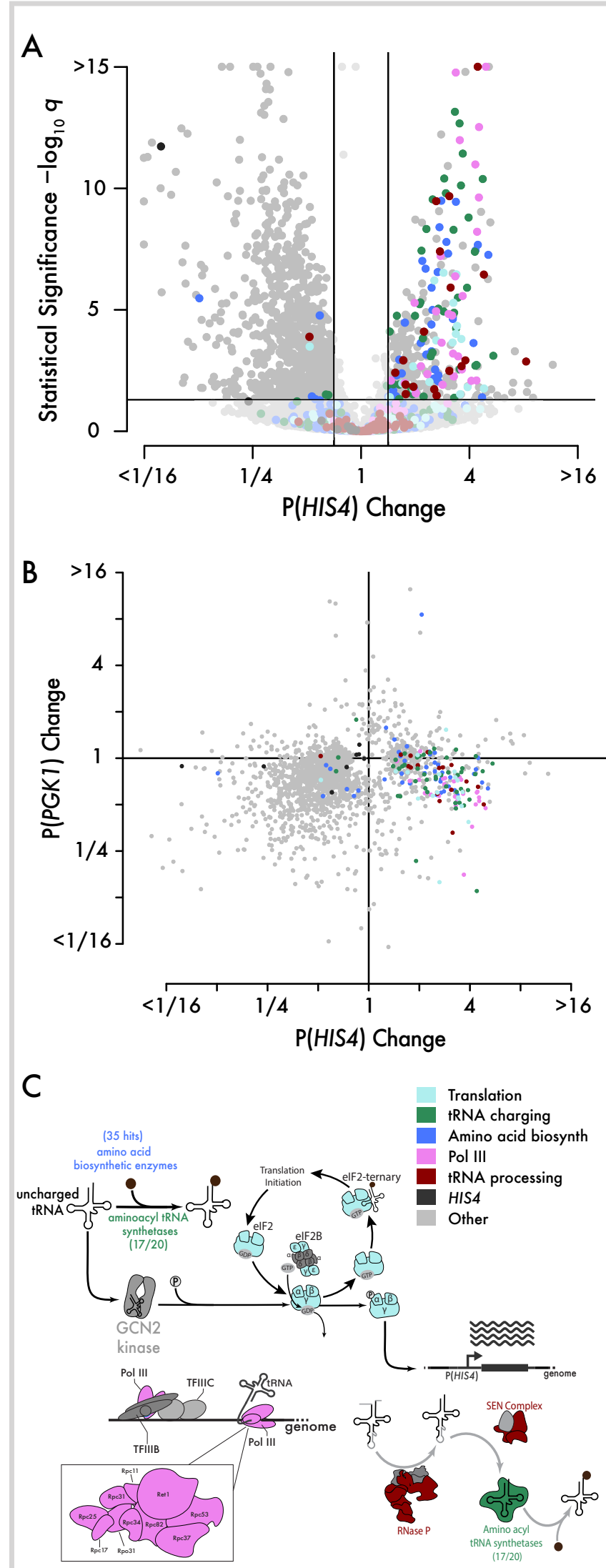

uncharged tRNAs accumulate. In order to distinguish ISR-mediated regulation from nonspecific or global effects on transcription, we also constructed a second guide library using the housekeeping promoter $P(P G K 1)$. CiBER-Seq profiling of $P$ (HIS4) induction identified 35 different guide RNAs targeting amino acid biosynthesis pathways, along with guides against 17 of the 20 of the aminoacyl-tRNA synthetases (Fig. 2, A and C), which all interfere with tRNA charging. We also observed P(HIS4) activation in response to knockdown of each individual component of the eIF2 translation initiation complex (Fig. 2, A and C); depletion of these proteins directly increases translation of the Gcn 4 transcription factor, leading to P(HIS4) induction $(16,20)$. All of these CRISPRi perturbations affected $P(H I S 4)$ specifically, and did not induce $P(P G K 1$ ) (Fig. 2B), although knockdown of general RNA polymerase II transcription reduced expression in both reporters (fig. S2, A through $\mathrm{C}$ ). In fact, $P(P G K 1)$ driven barcode expression was induced instead by knockdown of other glycolytic enzymes (fig. S2, A through C), suggesting a homeostatic transcriptional activation of $P G K 1$ expression in response to impaired glycolysis.

Surprisingly, we also found that overall tRNA depletion triggered the ISR transcriptional program. Guides targeting subunits of RNA polymerase III, which transcribes tRNAs, as well as the tRNA processing complex RNAse $P$ and the SEN tRNA splicing complex (21) all activated $P$ (HIS4) transcription (Fig. 2, A and C). In contrast to known ISR triggers, these genetic

Fig. 2. CiBER-seq recapitulates known genetic regulators of integrated stress response and identifies new regulators related to tRNA insufficiency. (A) Genome-wide CiBER-seq profile of $\mathrm{P}(H I S 4)$ transcription. Each point represents a different guide RNA, colored according to the function of the target gene. The lines indicate guides with a significant $(q<0.05)$ and substantial (>1.5-fold change) effect. (B) Comparison of CiBER-seq profiles of $\mathrm{P}(\mathrm{HIS} 4)$ and $\mathrm{P}(\mathrm{PGK1})$ transcription, colored as in (A). (C) Coverage of the known integrated stress response pathway, RNA polymerase III, and tRNA processing complexes by guides with significant effects on $P(H I S 4)$ activity. 
perturbations should not lead to the accumulation of uncharged tRNAs and in fact should reduce overall tRNA levels. While depletion of initiator methionyl-tRNA can induce $P(H I S 4)$ (22) and RNA polymerase III defects can lead to initiator methionyl-tRNA depletion (23), this effect cannot explain our observations here. Initiator tRNA does not contain an intron, and so the loss of SEN should not reduce initiator tRNA levels. To further exclude initiator $\mathrm{tRNA}$ depletion as an explanation for the effects of RNA polymerase III knockdown, we overexpressed initiator tRNA during CRISPRi-mediated ISR activation (fig. S2J and S2K). Impaired tRNA transcription showed no particular susceptibility to suppression of ISR response by high-copy overexpression of initiator $\mathrm{tRNA}$, relative to disruption of amino acid biosynthesis or tRNA charging (fig. S2K). Taken together, our results argue that elongator tRNA depletion can directly activate ISR transcription, perhaps through its effects on translation elongation.

\section{tRNA insufficiency triggers HIS4 transcription independently of elF2 $\alpha$ phosphorylation and Gcn2 kinase}

Because the integrated stress response is not activated during log-phase growth, our initial CiBER-seq data did not identify expression changes caused by loss of canonical, positive transducers of ISR signaling, such as GCN2 and GCN4. To uncover the phenotypes of these and other ISR pathway genes, we next looked for regulators whose depletion would block $P$ (HIS4) activation triggered by the toxic histidine analog 3-amino-1,2,4-triazole (3AT), which blocks histidine biosynthesis (Fig. 3, A and B). Guides that activate $P$ (HIS4) under replete nutrient conditions did not further elevate P(HIS4) expression upon 3AT treatment (Fig. 3B). We infer that Gcn4-mediated activation is saturated under these conditions, although we note that knockdown of Gcn4 degradation factors, such as PCL5 (24), can enhance 3AT-mediated P(HIS4) induction. Meanwhile, guides that reduced
$P$ (HIS4) transcription, including guides targeting the core RNA polymerase II transcription machinery (fig. S2, A, B, and C), did not interfere with $P$ (HIS4) activation upon 3AT treatment. We therefore reasoned that guides affecting amino acid biosynthesis, as well as tRNA charging, transcription, and processing, each saturated $\mathrm{P}($ HIS4) transcription through a shared, GCN4dependent mechanism. More broadly, these results illustrate the power of CiBER-Seq to dissect gene regulatory networks and identify novel interactors in even well-characterized systems.

Since one hallmark of ISR activation is the phosphorylation of eIF $2 \alpha$, we were curious whether tRNA insufficiency provoked this response. While CRISPRi knockdown of the amino acid biosynthetic enzyme ILV2 or the histidyl-tRNA synthetase HTS1 both induced eIF $2 \alpha$ phosphorylation, knock-down of the RNA polymerase III subunit RPC31 did not (Fig. 3C and 3D), consistent with previous observations (23). Deletion of GCN2, the only known eIF2 $\alpha$ kinase in yeast, completely blocked eIF $2 \alpha$ phosphorylation in all conditions tested (Fig. $3 \mathrm{C})$. Activation of $P(H I S 4)$ in the absence of eIF $2 \alpha$ phosphorylation further distinguished the response to $\mathrm{tRNA}$ depletion upon RPC31 knockdown and the classical ISR pathway.

We thus tested the genetic requirements for activation of endogenous HIS4 transcription in response to these CRISPRi-mediated perturbations. Deletion of GCN4 blocked HIS4 activation by RPC31 knockdown as well as ILV2 and HTS1 knockdown, and so the effects of tRNA insufficiency reflect a GCN4-mediated ISR. Strikingly, GCN2 deletion produced distinct effects across these three CRISPRi knockdowns (Fig. 3E). Deletion of GCN2 completely blocks HIS4 induction in response to ILV2 depletion, consistent with canonical models of ISR signaling (25). Although HTS1 knockdown triggers strong eIF2 $\alpha$ phosphorylation, GCN2 deletion only partially abrogated its transcriptional effects. Finally, RPC31 
bioRxiv preprint doi: https://doi.org/10.1101/2020.03.29.015057; this version posted March 31,2020. The copyright holder for this preprint (which was not certified by peer review) is the author/funder, who has granted bioRxiv a license to display the preprint in perpetuity. It is made available under aCC-BY 4.0 International license.

Muller et al.
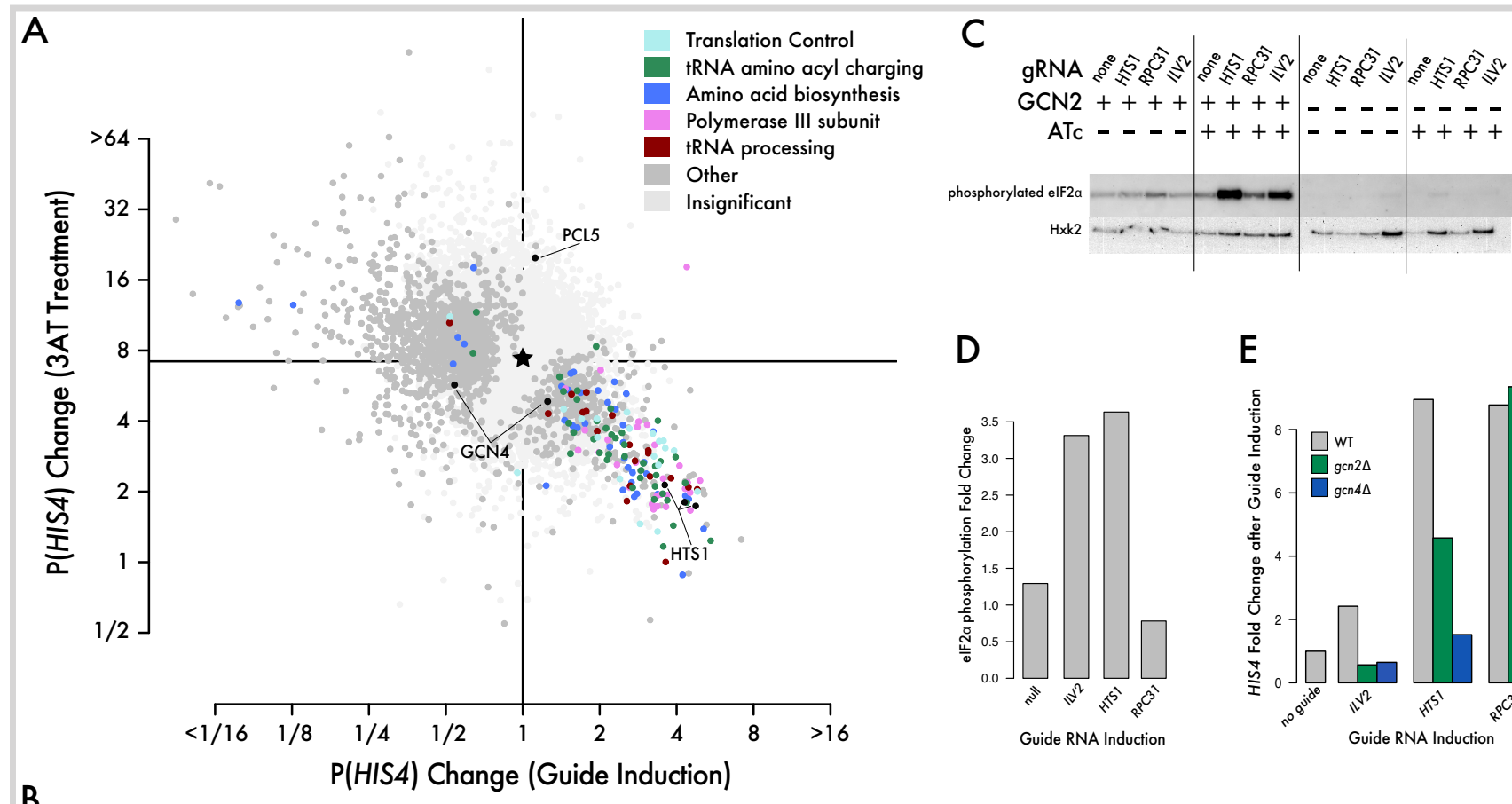

D
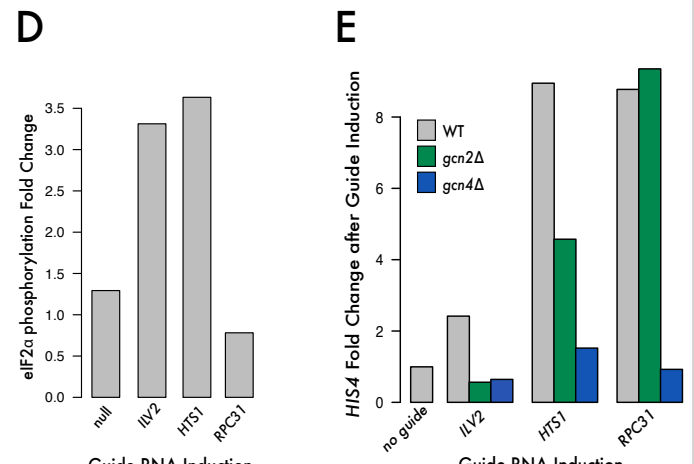

B
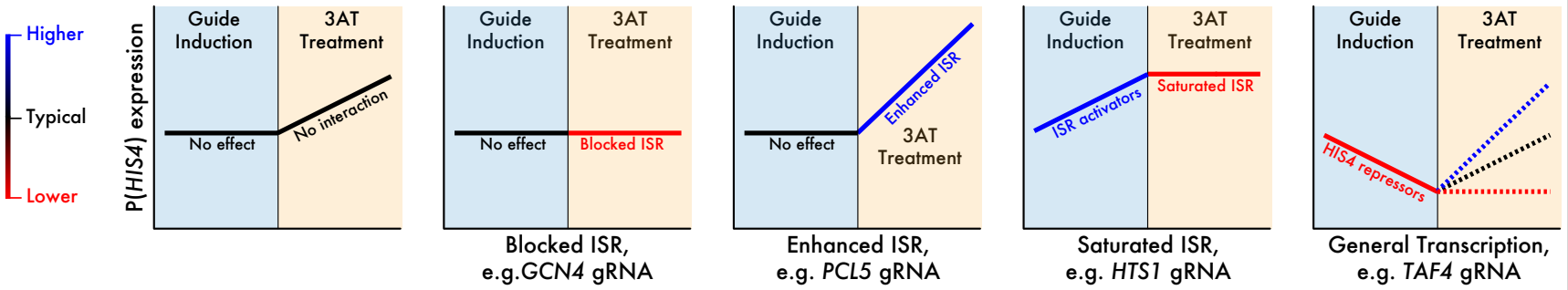

Fig. 3. tRNA insufficiency activates HIS4 transcription through a pathway that bypasses GCN2 kinase and elF2 $\alpha$ phosphorylation.(A) Comparison of P(HIS4) CiBER-seq profiles before and after 3AT treatment. (B) Schematic outlining observed ISR responses for functional categories of genes. (C) Western blot for eIF2 $\alpha$ phosphorylation. Knockdown of amino acid biosynthesis (ILV2 guide) and aminoacyl tRNA charging (HTS1 guide) results in eIF2 $\alpha$ phosphorylation while knockdown of RNA polymerase III (RPC31 guide) does not. GCN2 deletion abolishes eIF2 $\alpha$ phosphorylation. (D) Quantification of eIF2 $\alpha$ phosphorylation relative to hexokinase loading control in (C). (E) Endogenous HIS4 mRNA levels measured by qPCR. Endogenous HIS4 activation by ILV2, HTS1, or RPC31 knockdown is completely GCN4 dependent. HIS4 activation by ILV2 knockdown is also completely GCN2 dependent while effects of RPC31 knockdown are entirely GCN2 independent and HTS1 knockdown is only partially GCN2 dependent.

knockdown induces HIS4 transcription independent of GCN2, as we expected based on the lack of eIF $2 \alpha$ phosphorylation. These results suggest that translation elongation defects, arising from impaired tRNA recruitment, directly trigger GCN4-mediated transcriptional responses. Knockdown of tRNA synthetases activates the ISR by this pathway in parallel with the GCN2-dependent response to uncharged tRNAs.

\author{
Perturbations of the ARP2/3 complex prevent \\ ISR activation by HTS1 or RPC31 knockdown \\ Given that both Gen 2 activity and eIF2 $\alpha$ \\ phosphorylation were dispensable for ISR \\ activation in response to tRNA depletion, we \\ next sought to systematically identify genes \\ required for this response. We looked for genetic \\ perturbations that modified $P(H I S 4)$ responses to \\ different ISR triggers by performing dual-guide \\ CiBER-Seq, combining one guide that \\ individually activated the ISR with a second \\ guide from our genome-wide CRISPRi library to
}




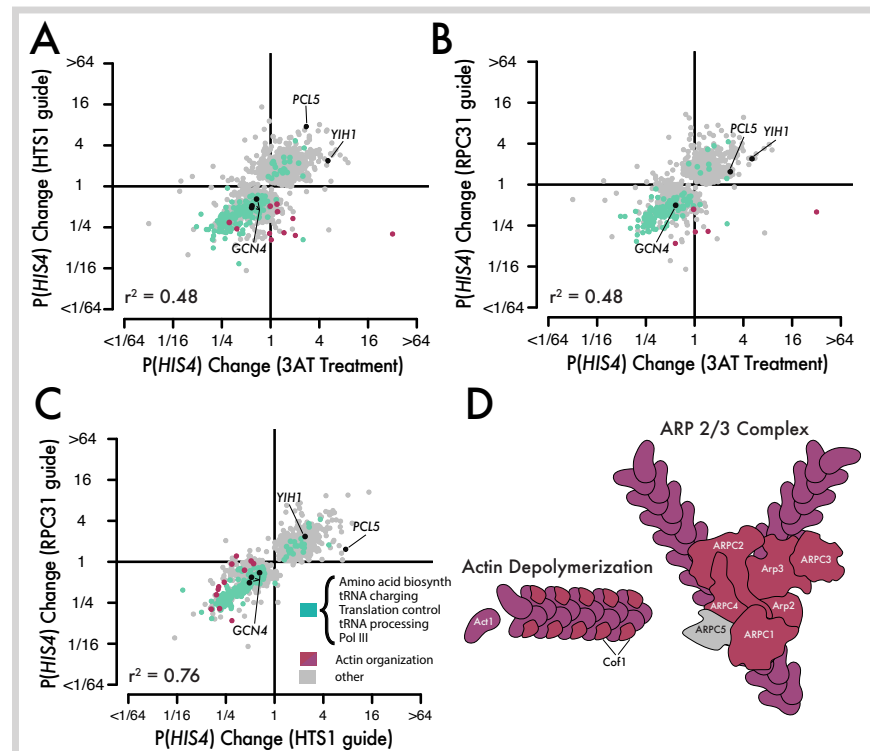

Fig. 4. Perturbations of the ARP2/3 complex prevent ISR activation by HTS 1 or RCP31 knockdown. (A to C) Pairwise comparison of $P(H I S 4)$ CiBER-seq profiles between ISR activation by 3AT treatment, HTS1 knockdown, and RPC31 knockdown. Each CiBER-seq profile was generated by measuring changes in P(HIS4) expression between the the guide in the context of an ISR activator relative to the change cause by the guide in isolation (Fig. 2A). (D) Coverage of actin cytoskeleton and ARP2/3 complex by guides that block ISR induction during HTS1 or RPC31 knockdown, but not 3AT treatment.

obtain quantitative, genome-wide genetic interaction profiles. We compared the interaction profiles of the histidyl-tRNA synthetase HTS1, which is required for tRNA charging, with those of the RNA polymerase III subunit RPC31, which is required for tRNA transcription (Fig. 4, A, B, and $\mathrm{C}$ ). We saw saturated $P$ (HIS4) induction after knockdown of HTS1 or RPC31, similar to the $P(H I S 4)$ saturation we observed upon 3AT treatment (Fig. 3A and $3 \mathrm{~B}$ ), as guides that activated the ISR on their own did not further enhance transcription in these dual-guide epistasis experiments. We also observed guides that had no effect on their own, but either suppressed ISR activation, such as guides against GCN4 itself, or enhanced the strength of the response, including guides against the degradation factor PCL5 or the ISR inhibitor YIH1 $(26,27)$.

We compared the genetic interaction profiles across three different ISR stimuli to test whether they were acting through similar or distinct pathways. Epistatic profiles of HTS1 and RPC31 knockdown resembled each other $\left(R^{2}=0.76\right)$ more closely than either resembled the profile during 3AT treatment $\left(R^{2}=0.48\right)$, although all three did overlap substantially. This pattern aligns with our observation that P(HIS4) activation by either tRNA charging defects or tRNA depletion are not completely GCN2 dependent, whereas the amino acid starvation response is fully GCN2 dependent (Fig. 3E). We thus looked for genetic interactions specific to these GCN2-independent responses and identified a surprising requirement for the actin cytoskeletal components in response to HTS1 and RPC31 knockdown (Fig. 4). Notably, knockdown of actin itself (ACT1) or genes encoding members of the Arp2/3 complex did not affect $\mathrm{P}(\mathrm{HIS} 4)$ transcription in normal growth or 3AT treatment (fig. S4A), but blocked $\mathrm{P}$ (HIS4) activation by tRNA charging defects or tRNA depletion (fig. S4, B and C). This could reflect interactions between the ISR inhibitor YIH1 and free actin monomers (27), or instead arise because of nuclear actin's role in transcription (28).

\section{Sumoylation is a post-translational regulator of Gcn4}

CiBER-Seq analysis of $P($ HIS4) regulation provided a comprehensive view of yeast ISR signaling, revealing distinct triggers for GCN4dependent transcriptional responses. However, this approach does not allow us to distinguish the different layers of Gcn4 regulation. Gcn4 abundance is controlled by regulated protein degradation (29) in addition to its wellcharacterized translational induction upon eIF $2 \alpha$ phosphorylation (16). Indeed, we saw epistatic enhancement of ISR activation from guides depleting the decay factor PCL5. We thus wanted to specifically analyze the regulators of Gcn 4 stability and translation, in isolation, and separate them from other effects on P(HIS4) activity. In order to couple these protein-level phenotypes with a transcriptional readout 
suitable for CiBER-seq, we returned to the synthetic, ZEM chimeric transcription factor used to initially validate barcode sequencing. Since barcode transcription is directly linked to the abundance of this synthetic transcription factor (Fig. 1B), we reasoned that it could couple protein-level regulation with expressed RNA barcode abundance (Fig. 5A).

We first profiled post-translational control of Gcn 4 by carrying out CiBER-Seq in yeast that constitutively express a protein fusion between Gcn 4 and the chimeric ZEM transcription factor (Fig. 5A). Levels of this transcription factor should reflect only post-translational regulation of Gcn4, and not its translational regulation, as it is expressed using the PGK1 promoter and 5' UTR. Knockdown of PCL5, which regulates Gcn4 degradation, affected the activity of the Gcn4ZEM post-translational reporter. Neither tRNA charging nor tRNA biogenesis had an effect, however. Instead, we found that disruption of sumoylation increased expression from $P(Z)$ (Fig. 5, B and C). We identified individually significant guide RNAs targeting nearly every step of the sumoylation pathway, including SUMO itself, SUMO-activating and SUMOconjugating enzymes, and the Siz1 SUMO ligase (30). Likewise, we identified guides against most components of the Nup84 subcomplex of the nuclear pore (31) that increased Gcn4-ZEM activity. This broad coverage of protein complex subunits and functionally related genes, here in the SUMOylation pathway and the Nup84 subcomplex (Fig. 5C), highlights the value of comprehensive, quantitative CiBER-Seq profiling. Sumoylation of Gcn4 has been shown to promote its eviction from chromatin, even when recruited through heterologous DNAbinding domains, as well as its subsequent degradation, both of which are expected to limit Gcn4-ZEM transactivation of barcoded reporters $(32,33)$. Knockdown of the SUMO conjugation machinery would thus relieve these limiting effects and enhance barcode expression. Elevated barcode expression during knockdown of the

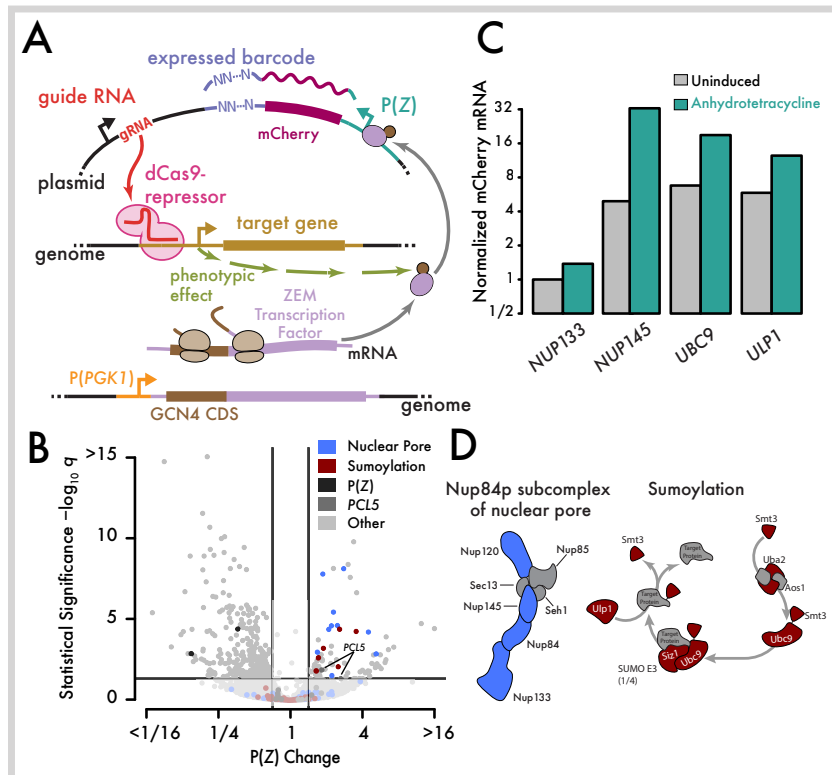

Fig. 5. Post-translational regulation of $\mathrm{Gcn} 4$ by sumoylation. (A) Schematic of indirect CiBER-seq experiment to identify post-translational regulators of Gcn4 (B) CiBER-seq response of Gcn4-ZEM / $P(Z)$ as in Fig. 2A. (C) Measurement of $m$ Cherry reporter mRNA abundance by qPCR. Knockdown of NUP133, NUP145, $U B C 9$, or ULP1 increase reporter mRNA levels.(D) Guides that activate $P(Z)$ with Gcn4-ZEM map to the Nup84 nuclear pore subcomplex and to the sumoylation pathway.

Ulp1 deconjugating protease could result from its role in SUMO maturation (34) or reflect a more complex requirement for a sumoylationdesumoylation cycle in Gcn4 regulation. The role of the nuclear pore is likely linked to sumoylation. In yeast, Ulp1 binds physically to the nuclear pore $(35,36)$, and mutations in the Nup84 complex can mimic some $u l p 1$ phenotypes through mislocalization (37) and reduction of Ulp1 activity (38). Furthermore, Ulp1 at the nuclear pore has been implicated directly in transcriptional activation (39).

\section{The GCN4 5' leader sequence is an intrinsic biosensor of translation stress}

We observed clear and coherent patterns of genetic perturbation affecting Gcn 4 protein activity, but no evidence that tRNA insufficiency affected its post-translational regulation. We next explored the translational control of GCN4, which results from regulatory upstream open reading frames in the GCN4 5' leader sequence 


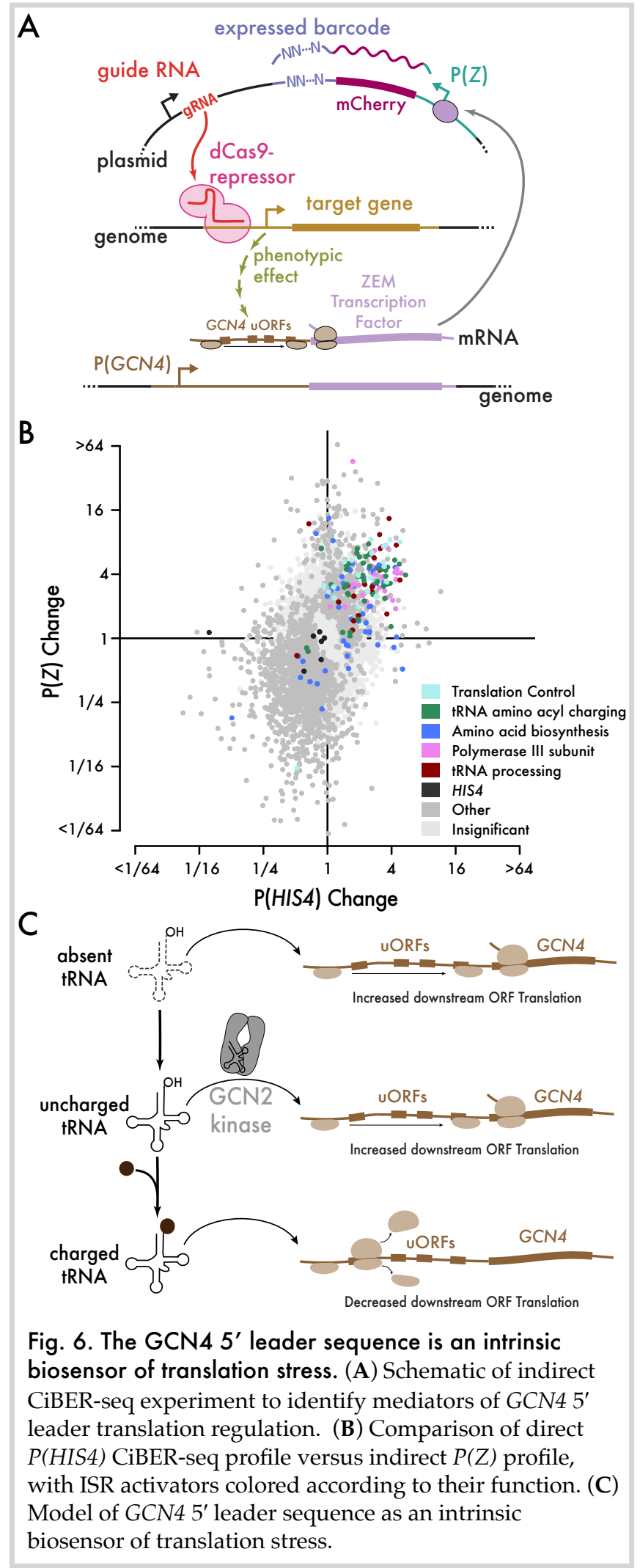

(16). In order to capture perturbations that affect this translation regulation, we drove expression of the ZEM synthetic transcription factor from the GCN4 promoter and 5' leader sequence and assessed guide effects on barcode expression (Fig. 6A).

The same guides that activated P(HIS4), including those that block tRNA biogenesis as well as tRNA charging, also increased $P(Z)$ in our CiBER-seq analysis of translational control through the GCN4 5' leader (Fig. 6B). By assessing this translational response in isolation, we observe effects occur in the same direction, but with larger magnitude, than $P$ (HIS4). This analysis supports the view that translational regulation is central to ISR activation, even when it proceeds independently of GCN2 and eIF2 $\alpha$ phosphorylation, although our data cannot exclude transcriptional effects mediated by the GCN4 promoter (40).

\section{DISCUSSION}

By linking CRISPR interference guides with barcoded expression reporters, we generate quantitative, genome-wide phenotypic profiles for specific molecular events in the cell. Our CiBER-seq approach allows us to address transcriptional, translational, and posttranslational regulation, enabling systematic genetic analysis of diverse biological processes. We leveraged quantitative CiBER-seq phenotypic profiles to gain new and surprising insights into the integrated stress response, identifying tRNA depletion as a previously unappreciated trigger for this well-characterized pathway.

Because translation is a resource-intensive biosynthetic process, most organisms sense translational stresses and respond with physiological changes that maintain homeostasis. In eukaryotes, amino acid starvation, which directly impacts tRNA charging and translation elongation, triggers a global decrease in translation initiation while also increasing transcription of amino acid biosynthesis genes (16). In many cases, reduced initiation is sufficient to restore normal 
elongation profiles (41). Indeed, when aminoacyl-tRNA synthetases are depleted, yeast reduce translation initiation until tRNA charging and utilization are balanced. Furthermore, in normal circumstances, these synthetases appear to buffer tRNA levels by sequestering uncharged tRNAs (42). Here, we find that the opposite situation - tRNA depletion in the presence of adequate synthetases and amino acids triggers a GCN2-independent ISR. Our comprehensive genetic data implicating many stages of tRNA biogenesis argue that GCN4 translation responds directly to elongator tRNA insufficiency, rather than depletion of initiator tRNA (23) or the accumulation of unprocessed nuclear tRNA precursors (43). As we have not identified other trans-acting regulatory pathways under these circumstances, we propose that the GCN4 5' leader is an intrinsic biosensor for translation elongation stress (Fig. 6C). Artificial stimuli, including tRNA depletion, can activate GCN4 translation independent of eIF $2 \alpha$ phosphorylation. In the natural history of budding yeast, the ISR likely evolved to sense nitrogen starvation and elicit general inhibition of translation through GCN2, along with GCN4dependent activation of homeostatic transcriptional programs (16). Our work thus expands the range of stresses known to activate the ISR and points to a general mechanism for sensing translational perturbations.

While we showed that the ISR is activated by artificial, genetic depletion of tRNAs, similar situations may arise in a natural context (44). Starvation and other stresses can induce tRNA cleavage $(45,46)$, and while much attention has focused on the positive roles of the resulting fragments (47), cleavage can also deplete the tRNA substrate and alter translation (48).

Effective tRNA depletion may also arise when tRNAs are sequestered in the nucleus and thus unavailable for cytosolic translation (21). Finally, an array of human disease mutations affecting tRNA biogenesis factors lead to neurodegenerative disorders, although the molecular basis for this effect is not clear $(44,49)$. Mutations in tRNA genes themselves can lead to tRNA insufficiency, ISR activation, and neurodegeneration, although in mammals this effect is GCN2 dependent $(50,51)$.

Our results demonstrate the power of CiBER-seq in elucidating the genetic architecture of regulation in the cell. We see comprehensive coverage of pathways and molecular complexes by guides targeting each individual component, indicating that our approach is both specific and sensitive. We identified ISR activation upon knockdown of each subunit of RNA polymerase III along with most proteins involved in tRNA processing, revealing tRNA depletion as the underlying trigger for the ISR. We identify other modes of regulation as well, such as sumoylation of Gcn4, and even detect the compensatory $P(P G K 1)$ activation in response to impaired glycolysis. Quantitative CiBER-seq profiles enable genetic interaction analysis that provides further insight into regulatory networks.

Correlated patterns of epistasis are a powerful tool for identifying genes that function together in pathways and complexes, and likewise chemogenomic comparison between chemical and genetic interaction profiles can reveal functional drug targets (11). Here, we leverage this advantage of CiBER-seq to identify a role for actin cytoskeletal components in ISR signaling. More broadly, our results highlight the power of CiBER-Seq to combine specific and quantitative molecular phenotypes with targeted genetic perturbations and thereby precisely dissect regulatory pathways. Indeed, a similar ReporterSeq technique was developed simultaneously with this work, and applied to elucidate known and novel pathways linking diverse stressors to the yeast heat shock response (52).

As the key components of CiBER-seq translate into nearly any organism, we anticipate many biological insights arising from broad application of our approach. 


\section{ACKNOWLEDGEMENTS}

We thank G. Brar, L. Lareau, E. Ünal, and members of the Ingolia and Lareau labs for discussion and advice. This work was funded by NIH grants DP2 CA195768 and R01 GM130996 (N.T.I.). This work used the Vincent J. Coates Genomics Sequencing Laboratory at UC Berkeley, supported by NIH instrumentation grant S10 OD018174.

\section{AUTHOR CONTRIBUTIONS}

R. M. and N. T. I. designed the experiments. R. M., Z. A. M, and L. F. carried out the experiments. R. M., L. F., and N. T. I. analyzed high-throughput sequencing data. R. M. and N. T. I. conceived the study and wrote the manuscript with input from all authors.

\section{COMPETING INTERESTS}

The authors declare no competing interests.

\section{DATA AND MATERIALS AVAILABILITY}

\begin{abstract}
All high-throughput sequencing data are available from the NCBI SRA under BioProject PRJNA578818. All source code is available at https: / / github.com/ingolia-lab/CiBER_seq. Plasmids and libraries will be made available through AddGene.
\end{abstract}

\section{REFERENCES}

1. G. J. Knott, J. A. Doudna, CRISPR-Cas guides the future of genetic engineering. Science 361, 866-869 (2018).

2. L. Cong et al., Multiplex genome engineering using CRISPR/Cas systems. Science 339, 819-823 (2013).

3. M. Jinek et al., RNA-programmed genome editing in human cells. Elife 2, e00471 (2013).

4. P. Mali et al., RNA-guided human genome engineering via Cas9. Science 339, 823-826 (2013).

5. L. A. Gilbert et al., CRISPR-mediated modular RNAguided regulation of transcription in eukaryotes. Cell 154, 442-451 (2013).

6. H. Koike-Yusa, Y. Li, E. P. Tan, C. Velasco-Herrera Mdel, K. Yusa, Genome-wide recessive genetic screening in mammalian cells with a lentiviral CRISPR-guide RNA library. Nat Biotechnol 32, 267-273 (2014).

7. O. Shalem et al., Genome-scale CRISPR-Cas9 knockout screening in human cells. Science 343, 84-87 (2014).
8. T. Wang, J. J. Wei, D. M. Sabatini, E. S. Lander, Genetic screens in human cells using the CRISPR-Cas9 system. Science 343, 80-84 (2014).

9. M. Kampmann, CRISPRi and CRISPRa Screens in Mammalian Cells for Precision Biology and Medicine. ACS Chem Biol 13, 406-416 (2018).

10. R. P. Patwardhan et al., High-resolution analysis of DNA regulatory elements by synthetic saturation mutagenesis. Nat Biotechnol 27, 1173-1175 (2009).

11. M. Breker, M. Schuldiner, Explorations in topologydelving underneath the surface of genetic interaction maps. Mol Biosyst 5, 1473-1481 (2009).

12. B. Adamson et al., A Multiplexed Single-Cell CRISPR Screening Platform Enables Systematic Dissection of the Unfolded Protein Response. Cell 167, 1867-1882 e1821 (2016).

13. A. Dixit et al., Perturb-Seq: Dissecting Molecular Circuits with Scalable Single-Cell RNA Profiling of Pooled Genetic Screens. Cell 167, 1853-1866 e1817 (2016).

14. D. A. Jaitin et al., Dissecting Immune Circuits by Linking CRISPR-Pooled Screens with Single-Cell RNA-Seq. Cell 167, 1883-1896 e1815 (2016).

15. P. Datlinger et al., Pooled CRISPR screening with single-cell transcriptome readout. Nat Methods 14, 297-301 (2017).

16. A. G. Hinnebusch, Translational regulation of GCN4 and the general amino acid control of yeast. Annu Rev Microbiol 59, 407-450 (2005).

17. A. Aranda-Diaz, K. Mace, I. Zuleta, P. Harrigan, H. ElSamad, Robust Synthetic Circuits for Two-Dimensional Control of Gene Expression in Yeast. ACS Synth Biol 6, 545-554 (2017).

18. N. J. McGlincy et al., A genome-scale CRISPR interference guide library enables comprehensive phenotypic profiling in yeast. bioRxiv. 10.1101 / 2020.03.11.988105 (2020).

19. J. D. Smith et al., Quantitative CRISPR interference screens in yeast identify chemical-genetic interactions and new rules for guide RNA design. Genome Biol 17, 45 (2016). 20. N. P. Williams, A. G. Hinnebusch, T. F. Donahue, Mutations in the structural genes for eukaryotic initiation factors 2 alpha and 2 beta of Saccharomyces cerevisiae disrupt translational control of GCN4 mRNA. Proc Natl Acad Sci U S A 86, 7515-7519 (1989).

21. A. K. Hopper, Transfer RNA post-transcriptional processing, turnover, and subcellular dynamics in the yeast Saccharomyces cerevisiae. Genetics 194, 43-67 (2013).

22. T. E. Dever, W. Yang, S. Astrom, A. S. Bystrom, A. G. Hinnebusch, Modulation of tRNA(iMet), eIF-2, and eIF-2B expression shows that GCN4 translation is inversely coupled to the level of eIF-2.GTP.Met-tRNA(iMet) ternary complexes. Mol Cell Biol 15, 6351-6363 (1995).

23. C. Conesa et al., Modulation of yeast genome expression in response to defective RNA polymerase IIIdependent transcription. Mol Cell Biol 25, 8631-8642 (2005). 
bioRxiv preprint doi: https://doi.org/10.1101/2020.03.29.015057; this version posted March 31,2020. The copyright holder for this preprint (which was not certified by peer review) is the author/funder, who has granted bioRxiv a license to display the preprint in perpetuity. It is made available under aCC-BY 4.0 International license.

Muller et al.

24. R. Shemer, A. Meimoun, T. Holtzman, D. Kornitzer, Regulation of the transcription factor Gcn 4 by Pho85 cyclin PCL5. Mol Cell Biol 22, 5395-5404 (2002).

25. N. S. Bae, A. P. Seberg, L. P. Carroll, M. J. Swanson, Identification of Genes in Saccharomyces cerevisiae that Are Haploinsufficient for Overcoming Amino Acid Starvation. G3 (Bethesda) 7, 1061-1084 (2017).

26. H. Kubota, Y. Sakaki, T. Ito, GI domain-mediated association of the eukaryotic initiation factor 2alpha kinase GCN2 with its activator GCN1 is required for general amino acid control in budding yeast. J Biol Chem 275, 20243-20246 (2000).

27. E. Sattlegger et al., YIH1 is an actin-binding protein that inhibits protein kinase GCN2 and impairs general amino acid control when overexpressed. J Biol Chem 279, 29952-29962 (2004).

28. I. A. Olave, S. L. Reck-Peterson, G. R. Crabtree, Nuclear actin and actin-related proteins in chromatin remodeling. Annu Rev Biochem 71, 755-781 (2002).

29. D. Kornitzer, B. Raboy, R. G. Kulka, G. R. Fink, Regulated degradation of the transcription factor Gcn4. EMBO J 13, 6021-6030 (1994).

30. E. S. Johnson, Protein modification by SUMO. Annu Rev Biochem 73, 355-382 (2004).

31. A. Hoelz, E. W. Debler, G. Blobel, The structure of the nuclear pore complex. Annu Rev Biochem 80, 613-643 (2011).

32. E. Rosonina, S. M. Duncan, J. L. Manley, Sumoylation of transcription factor Gcn4 facilitates its Srb10-mediated clearance from promoters in yeast. Genes Dev 26, 350-355 (2012).

33. A. Akhter, E. Rosonina, Chromatin Association of Gcn 4 Is Limited by Post-translational Modifications Triggered by its DNA-Binding in Saccharomyces cerevisiae. Genetics 204, 1433-1445 (2016).

34. S. J. Li, M. Hochstrasser, A new protease required for cell-cycle progression in yeast. Nature 398, 246-251 (1999).

35. S. J. Li, M. Hochstrasser, The Ulp1 SUMO isopeptidase: distinct domains required for viability, nuclear envelope localization, and substrate specificity. J Cell Biol 160, 1069-1081 (2003).

36. V. G. Panse, B. Kuster, T. Gerstberger, E. Hurt, Unconventional tethering of Ulp1 to the transport channel of the nuclear pore complex by karyopherins. Nat Cell Biol 5, 21-27 (2003).

37. B. Palancade et al., Nucleoporins prevent DNA damage accumulation by modulating Ulp1-dependent sumoylation processes. Mol Biol Cell 18, 2912-2923 (2007).

38. J. O. Rouviere et al., A SUMO-dependent feedback loop senses and controls the biogenesis of nuclear pore subunits. Nat Commun 9, 1665 (2018).

39. L. Texari et al., The nuclear pore regulates GAL1 gene transcription by controlling the localization of the SUMO protease Ulp1. Mol Cell 51, 807-818 (2013).

40. G. Albrecht, H. U. Mosch, B. Hoffmann, U. Reusser, G. $\mathrm{H}$. Braus, Monitoring the Gcn 4 protein-mediated response in the yeast Saccharomyces cerevisiae. J Biol Chem 273, 12696-12702 (1998).

41. A. M. Darnell, A. R. Subramaniam, E. K. O'Shea, Translational Control through Differential Ribosome Pausing during Amino Acid Limitation in Mammalian Cells. Mol Cell 71, 229-243 e211 (2018).

42. M. R. McFarland et al., The molecular aetiology of tRNA synthetase depletion: induction of a GCN4 amino acid starvation response despite homeostatic maintenance of charged tRNA levels. Nucleic Acids Res, (2020).

43. H. Qiu et al., Defects in tRNA processing and nuclear export induce GCN4 translation independently of phosphorylation of the alpha subunit of eukaryotic translation initiation factor 2. Mol Cell Biol 20, 2505-2516 (2000).

44. S. Kirchner, Z. Ignatova, Emerging roles of tRNA in adaptive translation, signalling dynamics and disease. Nat Rev Genet 16, 98-112 (2015).

45. S. R. Lee, K. Collins, Starvation-induced cleavage of the tRNA anticodon loop in Tetrahymena thermophila. J Biol Chem 280, 42744-42749 (2005).

46. D. M. Thompson, C. Lu, P. J. Green, R. Parker, tRNA cleavage is a conserved response to oxidative stress in eukaryotes. RNA 14, 2095-2103 (2008).

47. S. Yamasaki, P. Ivanov, G. F. Hu, P. Anderson, Angiogenin cleaves tRNA and promotes stress-induced translational repression. J Cell Biol 185, 35-42 (2009).

48. D. Huh et al., An adaptive stress-induced tRNA depletion response mediates codon-based gene repression and growth suppression. bioRxiv, 416727 (2018).

49. M. Kapur, C. E. Monaghan, S. L. Ackerman, Regulation of mRNA Translation in Neurons-A Matter of Life and Death. Neuron 96, 616-637 (2017).

50. R. Ishimura et al., RNA function. Ribosome stalling induced by mutation of a CNS-specific tRNA causes neurodegeneration. Science 345, 455-459 (2014).

51. R. Ishimura, G. Nagy, I. Dotu, J. H. Chuang, S. L. Ackerman, Activation of GCN2 kinase by ribosome stalling links translation elongation with translation initiation. Elife 5, (2016).

52. B. D. Alford, G. Valiant, O. Brandman, Genome-wide, time-sensitive interrogation of the heat shock response under diverse stressors via ReporterSeq. bioRxiv. 10.1101/2020.03.29.014845 (2020).

53. N. J. McGlincy et al., A genome-scale CRISPR interference guide library enables comprehensive phenotypic profiling in yeast. bioRxiv, 2020.2003.2011.988105 (2020).

54. M. A. Sheff, K. S. Thorn, Optimized cassettes for fluorescent protein tagging in Saccharomyces cerevisiae. Yeast 21, 661-670 (2004).

55. V. Stovicek, G. M. Borja, J. Forster, I. Borodina, EasyClone 2.0: expanded toolkit of integrative vectors for stable gene expression in industrial Saccharomyces cerevisiae strains. J Ind Microbiol Biotechnol 42, 1519-1531 (2015). 
56. T. W. Christianson, R. S. Sikorski, M. Dante, J. H. Shero, P. Hieter, Multifunctional yeast high-copy-number shuttle vectors. Gene 110, 119-122 (1992).

57. R. D. Gietz, R. H. Schiestl, High-efficiency yeast transformation using the LiAc/SS carrier DNA/PEG method. Nat Protoc 2, 31-34 (2007).

58. M. Ares, Isolation of total RNA from yeast cell cultures. Cold Spring Harb Protoc 2012, 1082-1086 (2012).

59. A. M. McGeachy, Z. A. Meacham, N. T. Ingolia, An Accessible Continuous-Culture Turbidostat for Pooled Analysis of Complex Libraries. ACS Synth Biol 8, 844-856 (2019).

60. L. Myint, D. G. Avramopoulos, L. A. Goff, K. D. Hansen, Linear models enable powerful differential activity analysis in massively parallel reporter assays. BMC Genomics 20, 209 (2019). 


\section{MATERIALS AND METHODS}

\section{Plasmid materials}

pRS416-dCas9-Mxi1 + TetR + pRPR1(TetO)-NotIgRNA was a gift from Ronald Davis (Addgene plasmid \# 73796). pKT0139 was a gift from Kurt Thorn (Addgene plasmid \# 8731). pHES836 was a gift from Hana El-Samad (Addgene plasmid \# 89195). pHES795 was a gift from Hana El-Samad (Addgene plasmid \# 87943). pCfB2337 was a gift from Irina Borodina (Addgene plasmid \# 67555). pCfB2226 was a gift from Irina Borodina (Addgene plasmid \# 67533). pCfB2189 was a gift from Irina Borodina (Addgene plasmid \# 67532).

\section{Plasmid construction}

Plasmid assembly was carried out using standard molecular biology techniques as described below, and verified by Sanger sequencing. All PCR reactions were performed using Q5 polymerase (NEB M0491S) according to manufacturer protocols. Restriction enzymes were obtained from NEB and high-fidelity (HF) variants were used when available. DNA fragments were size-selected by gel electrophoresis in 1\% agarose. Gibson assembly reactions were carried out using NEBuilder HiFi DNA Assembly Master Mix (NEB E2621L) with DNA fragments containing homology arms between 15 and 20 base pairs long. DNA was purified and concentrated as necessary with DNA Clean \& Concentrator (Zymo D4013). Transformations were performed in Stbl3 chemically competent cells provided by the QB3 Berkeley Macrolab facility and plated on appropriate antibiotic plates for colony selection. Plasmid DNA was purified from liquid cultures using DNA miniprep kit (NEB \#T1010).

pNTI660 was constructed in several steps. First, pNTI601 (pRS416-dCas9-Mxi1 + TetR + pRPR1(TetO)-NotI-gRNA, AddGene \# 73796) (19) was amplified with NM717 and NM724 (53) to isolate the gRNA expression cassette and the resulting PCR product was re-circularized by Gibson assembly to create pNTI646. pNTI646 was then digested with MfeI and combined with oligonucleotide NI1025 to create pNTI660.

pNTI725 was created in several steps. First, the promoter $P(P G K 1)$ was amplified from $S$. cerevisiae BY4741 genomic DNA using NI553 and NI554, and the resulting product was subcloned and used as a template for $P(P G K 1)$ amplification. Yeast-optimized yECitrine coding sequence was amplified from pNTI189 (pKT0139 AddGene \#8731) (54) using primers RM151 and RM155 and cloned downstream of $P$ (PGK1). Next, the $P(P G K 1)$-yECitrine expression fragment was isolated by digestion with SacI and BsrgI restriction sites, and pNTI660 was linearized by digestion with MfeI. Doublestranded DNA splints were created by annealing oligonucleotides RM317 and RM318, and oligonucleotides RM319 and RM320. Digested $P(P G K 1)$-yECitrine and pNTI660 were joined together using these splints in a four-piece Gibson assembly reaction. The NotI site for gRNA insertion was replaced with an NruI site by digesting the backbone with NotI and then introducing single-stranded oligonucleotide RM321 by Gibson assembly. Finally, the Illumina TruSeq Read1 site was added to the beginning of the terminator $T(A D H 1)$ by annealing singlestranded oligonucleotides RM323 and RM324, extending them using Q5 polymerase, digesting the backbone with AscI and introducing the double-stranded product into the digested plasmid by Gibson assembly.

pNTI726 was constructed by replacing $P$ (PGK1) in pNTI725 with estradiol-responsive $\mathrm{P}(G A L 1)$. $\mathrm{P}($ GAL1) with an embedded Zif268 binding site was PCR amplified from pHES836 using primers RM348 and RM349. pNTI725 was digested with SacI and EcoRI and amplified P(GAL1) was then Gibson assembled into the backbone.

pNTI727 was generated by inserting the gRNA sequence targeting $P(Z)$ into pNTI726. Plasmid pNTI726 was digested with NruI, singlestranded oligonucleotides RM389 and RM390 were annealed, extended using Q5 DNA polymerase, and the double-stranded product 
was introduced into the linearized plasmid by Gibson assembly.

pNTI728 was generated by inserting the gRNA sequence targeting $P(A D H 1)$ into pNTI726, using the same methods as pNTI727, using oligonucleotides RM383 and RM384.

pNTI729 was constructed by first amplifying the ZEM transcription factor from pNTI638 (pHES795 Addgene \#87943) (17) in two pieces, using oligonucleotide primers RM352 with RM353 and RM354 with RM355. The easy clone vector pCfB2337 (Addgene \#67555) (55) was then digested with HindIII and the three fragments were combined by Gibson assembly.

pNTI730 was constructed by replacing the promoter $P(A D H 1)$ in pNTI729 with the $P(P G K 1)$ promoter. pNTI729 was digested with PvuII and NheI, P(PGK1) was amplified from pNTI725 using primers RM417 and RM418, and the two fragments were combined by Gibson assembly.

pNTI731 through pNTI737, expressing guide RNAs against ILV2, HTS1, RPC31, NUP133, NUP145, ULP1, and UBC9 respectively, were subcloned into the integrating plasmid pCfB2189. First, DNA fragments containing the guide RNA expression cassette were amplified from the pool of gRNA plasmids using forward primers RM639, RM517, RM518, and RM641 through 644, respectively, that anneal to the unique nucleotide barcode sequence, and a reverse primer RM519 that binds downstream of the gRNA scaffold. Fragments were amplified with Gibson homology arms on each end. The integration vector pCfB2189 (Addgene \#67532)

(55) was digested with HindIII and gRNAexpressing fragments were transferred into this backbone by Gibson assembly.

pNTI738 for initiator methionyl-tRNA overexpression was constructed by amplifying the initiator methionyl-tRNA locus IMT4 from yeast genomic DNA using primers RM636 and RM637, digesting the high-copy yeast plasmid pRS426 (ATCC \#77107) (56) with EcoRI, and combining them by Gibson assembly.
pNTI739 was constructed from pNTI730. The GCN4 CDS was amplified from yeast genomic DNA using primers RM515 and RM516 and inserted upstream of, and in frame with, the synthetic transcription factor by digesting pNTI730 with NheI and introducing GCN4 by Gibson assembly.

pNTI740 was constructed by digesting NTI730 with PvuII and NheI, amplifying the GCN4 promoter and 5 UTR from yeast genomic DNA using primers RM513 and RM514, and combining the two fragments by Gibson assembly.

pNTI741, the P(HIS4)-yECitrine PCR template used for downstream CiBER-seq plasmid library preparation, was generated by replacing the $P(P G K 1)$ in pNTI725 with $P$ (HIS4). pNTI725 was digested with SacI and NheI, P(HIS4) was amplified from yeast genomic DNA using primers RM499 and RM500, and the two fragments were combined by Gibson assembly.

pNTI742 was made in several steps. First, the NotI restriction site of pNTI660 was replaced with an AvrII restriction site by digesting with NotI and introducing a replacement cassette, comprising annealed oligonucleotides RM459 and RM460, by Gibson assembly. Next, the vector was digested with SacI and MfeI. The $T(A D H 1)$ terminator and TruSeq $\mathrm{R} 1$ priming site were amplified from $\mathrm{pNTI726}$ using primers RM489, RM490, and RM491, in a nested PCR approach where RM490 was used at $0.1 x$ the normal concentration. The resulting PCR product was joined with the digested vector by Gibson assembly.

\section{Plasmid library construction}

All pooled plasmid libraries were constructed by Gibson-style assembly using HiFi DNA assembly master mix (NEB E2621L) and transformed using either ElectroMAX DH10B (ThermoFisher \#18290015) or NEB® 10-beta Competent E. coli (NEB \#C3019H) according to manufacturer protocol. All column purifications were performed using Zymo DNA Clean and 
Concentrator-5 (Zymo \#D4013). Dilutions of reach transformation were plated to estimate library size and ensure sufficiency library diversity. Plasmid libraries were harvested from liquid culture using the Monarch ${ }^{\circledR}$ Plasmid DNA Miniprep Kit (NEB \#T1010). Miniprep kit reagents were scaled to accommodate one spin column for every $5 \mathrm{~mL}$ of culture.

To generate estradiol-inducible barcodeexpressed plasmid libraries (Fig1), pNTI726 and pNTI728 were first digested with BamHI. Five barcodes were generated by annealing primer RM396 with one of five barcode primers, RM391, RM392, RM393, RM394, or RM399, and extending the annealed duplex using Q5 polymerase. Four of these barcodes were inserted by Gibson assembly into BamHIlinearized pNTI726 and the last barcode DNA fragment inserted into BamHI-linearized pNTI728. Gibson reactions were purified and concentrated, transformed into ElectroMAX DH10B cells, and inoculated directly into $200 \mathrm{ml}$ of LB-carbenicillin media. Pooled libraries were harvested when batch liquid culture reached OD of 4 .

To generate gRNA plasmid libraries, 100 pg of CRISPRi guide RNA oligonucleotide library was amplified by PCR using Q5 polymerase using primers NM636 and NM637 (53). pNTI742 was digested with AvrII and the amplified guide fragments were introduced into the digested vector by Gibson assembly. The assembly product was purified and transformed into ElectroMAX DH10B cells by electroporation. Electroporated cells were inoculated directly into $500 \mathrm{~mL}$ of LB-carbenicillin media and harvested at an OD of 2. Serial dilutions of the initial transformation were plated to ensure sufficient library diversity ( $>50 x$ coverage of 60,000 guides). Plasmid library was purified using NEB miniprep kit and pooled library was analyzed by Sanger sequencing.

Barcodes were then added to the gRNA plasmid library, targeting an average of four barcodes per guide RNA (240,000 barcodes in total). First, barcode DNA fragments were generated by annealing oligos RM504 and RM505 and extending the duplex using Q5 polymerase. The following thermocycler conditions were used to avoid amplification of any specific barcode sequence. Initial denaturation at $98^{\circ} \mathrm{C}$ for $45 \mathrm{~s}$, six cycles of annealing and extension at $68^{\circ} \mathrm{C}$ for $15 \mathrm{~s}$ and $72{ }^{\circ} \mathrm{C}$ for 5 s respectively, and finally a $12{ }^{\circ} \mathrm{C}$ hold. The barcode fragments were digested with BamHI and MfeI in order to eliminate barcode fragments that contain these restriction sites and then purified using a DNA Clean and Concentrator-5. The library of gRNA-expressing plasmids was then digested with AflII and the barcode DNA fragments were introduced by Gibson assembly. The assembly reaction was purified using a DNA Clean and Concentrator-5 then transformed into NEB® 10-beta Competent E. coli (High Efficiency) according to manufacturer's protocol. Transformation dilutions were plated to estimate total number of transformants, and in parallel inoculated at several dilutions directly into LB-carbenicillin media. Liquid culture corresponding to roughly 240,000 transformants was harvested at an OD of 2 and plasmid library was extracted using NEB miniprep kit. Reagents were scaled to accommodate one spin column for every $5 \mathrm{~mL}$ of culture. This barcoded gRNA library was pairedend sequenced (see "Barcode to gRNA assignment" section) to assign barcodes with guide RNAs.

$P$ (HIS4)-yECitrine was amplified from pNTI741 using primers RM501 and RM502, P(PGK1)yECitrine was amplified from pNTI725 using primers RM501 and RM503, and two versions of estradiol-inducible $\mathrm{P}(Z)$-mCherry with unique nucleotide identifiers were amplified with either RM522 and RM524 or RM523 and RM524. The barcoded gRNA plasmid library was digested with BamHI and the four PCR products were introduced into the digested library by Gibson assembly to create the four CiBER-seq libraries. These libraries were then transformed into ElectroMAX DH10B cells by electroporation. 
Electroporated cells were inoculated directly into $500 \mathrm{~mL}$ of LB-carbenicillin media and harvested at an OD of 2. Serial dilutions of the initial transformation were plated to ensure sufficient library diversity ( $>50$ x coverage of 60,000 guides). Plasmid library was purified using NEB miniprep kit and pooled library was analyzed by Sanger sequencing.

\section{Construction of yeast strains}

All yeast strains were constructed by transforming PCR products or plasmids linearized by restriction enzyme digestion into yeast by standard lithium acetate transformation (57). Selected, clonal transformants were confirmed by two genotyping PCRs, using primer pairs that amplify across the junction on either side of the integration. Genotyping reactions were performed with OneTaq Hotstart Polymerase (NEB \#M0481S).

Yeast strains used in this study are listed in Table 3. Strains with $g c n 2 \Delta$ or $g c n 4 \Delta$ genotype were constructed by first amplifying the hygromycin resistance cassette from $\mathrm{pNTI729}$ by PCR, with flanking sequence homologous to the $5^{\prime}$ and $3^{\prime}$ ends of the GCN2 or GCN4 coding sequence, using primers RM579 through RM582 for $g c n 2 \Delta$ and primers RM583 through RM586 for gcn4 $\Delta$. Primers RM589 and RM590 were used to genotype the upstream junction for $g c n 2 \Delta$ while RM591 and RM592 were used for the downstream junction. Primers RM593 and RM590 were used to genotype the upstream junction for gcn4 $\Delta$ while RM591 and RM594 were used for the downstream junction.

Guide RNA expression cassettes were integrated using vectors from the EasyClone 2.0 toolkit for yeast genomic integration (55). Plasmids using backbone pCfB2189 were integrated by digesting with NotI, transforming this digestion, and selecting transformants on SCD -Leu plates. Plasmids using backbone pCfB2337 were integrated by digesting with NotI, transforming this digestion, plating transformations on nonselective YEPD media overnight, and then replica plating on hygromycin plates. Histidine prototrophy was restored by digesting pCfB2226 with NotI, transforming into the appropriate strain, and selecting transformants on SCD -His plates. Plasmid pNTI647, expressing dCas9 and tetR, was integrated as described in (53). Integration of guide RNA expression cassettes was validated by amplifying across the upstream junction with RM527 and RM373 and across the downstream junction with RM374 and RM528. Integration of synthetic transcription factor variants were genotyped by amplifying across the upstream junction with RM372 and RM373 and across the downstream junction with RM374 and RM375.

\section{Media}

LB media was prepared by dissolving LB medium capsules (MP Biomedicals \#3002-31) in ultra-pure water and sterilized by autoclaving, according to manufacturer instructions. LB Media was supplemented with $50 \mu \mathrm{g} / \mathrm{ml}$ carbenicillin (Sigma \#C1389) for antibiotic selection. YEPD was prepared by dissolving yeast extract (RPI \# Y20026) and peptone (RPI \#20241) in ultra-pure water, sterilizing by autoclaving, then supplementing with $2 \%$ final concentration of sterilized dextrose (Fisher Chemical \#D16-500). Synthetic complete dropout (SCD) media was prepared by dissolving Yeast Nitrogen Base (BD Difco \#291940), dextrose to $2 \%$ final concentration, and the appropriate drop-out mix in ultra-pure water, then sterilizing by filtration. SCD -Leu drop-out media was prepared using Drop-out Mix Synthetic Minus Leucine (US Biological \#D9525), SCD -Ura drop-out media was prepared using Drop-out Mix Synthetic Minus Uracil (US Biological \# D9535).

\section{qRT-PCR}

RNA was isolated from yeast by acid phenol extraction (58). Reverse transcription was carried out using Protoscript II reverse transcriptase (NEB \#M0368L) with oligo-dT priming according to manufacturer instructions. Quantitative RT-PCR was carried out using DyNAmo qPCR mastermix (ThermoFisher 
\#F410L) according to manufacturer protocols, using a CFX96 Touch Real-Time PCR Detection System. Primers, provided in Table 5, were designed using Primer Blast and validated by using a cDNA dilution standard curve.

\section{Fluorescence measurements}

Expression of yECitrine, under the control of the ZEM transcription factor, (Fig. 1) was monitored using a 96-well plate reader (Tecan SPARK® Multimode Microplate Reader). Overnight cultures were back-diluted in 96-well round bottom plates to OD 0.05 in SCD -Ura selective media containing beta-estradiol and / or anhydrotetracycline as indicated. Fluorescence (excitation at $516 \mathrm{~nm}$ and emission at $540 \mathrm{~nm}$ ) and $\mathrm{OD}_{600}$ was measured in triplicate every 15 minutes.

\section{Turbidostat continuous culture}

Yeast populations transformed with plasmid libraries were inoculated into a custom turbidostat (59) and maintained in SCD -Ura media at a density corresponding to $\mathrm{OD}_{600}$ roughly 0.8 . When growth rate reached a steady state (corresponding to a doubling time of roughly 90 minutes), a $50 \mathrm{ml}$ pre-induction sample was collected. Guide RNA expression was then induced by injecting concentrated anyhydrotetracycline into both the growth chamber and turbidostat media reservoir to obtain a final concentration of $250 \mathrm{ng} / \mathrm{ml}$ of anyhydrotetracycline. Six doublings later (9 hours) a $50 \mathrm{~mL}$ post-induction sample was collected. For 3AT treatment, samples were induced by injecting concentrated 3AT into both the growth chamber and turbidostat media reservoir to obtain a final concentration of 90 mM 3AT. Two hours later, a post-induction sample was collected. For CiBER-seq experiments involving the ZEM transcription factor, media was supplemented with $8 \mathrm{nM}$ betaestradiol throughout the course of the experiment. Collected samples were pelleted by centrifugation at $4,000 \times g$ for 5 minutes, the media was aspirated, and the pellets were stored at $-80^{\circ} \mathrm{C}$.

\section{Barcode sequencing}

All PCR reactions were performed using Q5 polymerase (NEB M0491S) according to manufacturer protocols. PCR cycle numbers were adjusted as needed (between 6-12 cycles) to obtain adequate concentration of product for sequencing, while avoiding overamplification. DNA was purified using DNA Clean \& Concentrator (Zymo \#D4013) according to the manufacturer instructions. When necessary, AMPure XP beads (Beckman Coulter \# A63881) were used to purify full-length DNA product. Size distributions and concentrations of the sequencing libraries were measured before sequencing using an Agilent TapeStation 2200 and High Sensitivity D1000 ScreenTape. To ensure proper clustering on the Illumina HiSeq4000, CiBER-seq libraries were either pooled with RNA-seq libraries and standard 2\% PhiX, or individually with $10 \%$ PhiX. PCR products were pooled and sequenced on HISeq 4000 SR50 (Vincent J Coates Sequencing facility). Sequencing data accession numbers are available in Table 7.

\section{DNA library preparation}

Plasmid DNA was extracted from yeast pellets using Zymo yeast miniprep kit (Zymo \# D2004) according to manufacturer protocol, except as described below. Reagent volumes were scaled to accommodate roughly 250 million cells, as yeast pellets included $25 \mathrm{OD}_{600} \mathrm{ml}$ of yeast. Zymolyase concentration was increased to $1 \mu \mathrm{L}$ for every 10 million cells and the digestion time was doubled to ensure complete cell wall digestion.

Extracted barcode expression plasmid libraries derived from pNTI726 and pNTI728 backbones (Fig. 1) were prepared by PCR amplification of barcode sequences with primers that incorporated flanking TruSeq adapters. Barcode sequences were first amplified by PCR with RM411 and an i5 primer from NEBNext ${ }^{\circledR}$ Multiplex Oligos (NEB \#E7600S) for 12 cycles. PCR products were purified and concentrated using a DNA Clean and Concentrator-5, and 
then used as the template for an additional 6 cycles of PCR amplification with NEBNext i5 and i7 primers (NEB \#E7600S).

To achieve a more linear amplification, all other CiBER-seq DNA libraries were prepared using an initial linear amplification by in vitro transcription. Extracted plasmid was linearized by restriction digestion with MfeI for P(PGK1) and $P$ (HIS4) plasmid libraries (Fig. 2 through 4 ) or PvuII for $P(Z)$ libraries (Fig. 5 and 6). The digestion product was purified using a DNA Clean and Concentrator-5, and used as the template for an IVT reaction using T7 HiScribe (NEB \#E2040S) according to the manufacturer protocol for short transcripts, with an overnight incubation at $37^{\circ} \mathrm{C}$. The reaction was then treated with DNase I to remove template DNA and RNA product was purified using an RNA Clean and Concentrator-5 (Zymo \#R1016). Purified RNA was used as the template for reverse transcription using ProtoScript II according to manufacturer protocol, using sequence-specific primer RM511 for $P(P G K 1)$ and $P$ (HIS4) plasmid libraries (Fig. 2 through 4) or RM546 for $P(Z)$ libraries (Fig. 5 and 6). Reverse transcription cDNA product was treated for 30 mins with 0.5 $\mu$ L RNAse A (ThermoFisher \#EN0531) and 0.5 $\mu \mathrm{L}$ RNAse H (NEB \# M0297S) to remove RNA, and then DNA was purified using a DNA Clean and Concentrator-5 column. The sequencespecific reverse transcription primers RM511 and RM546 incorporate the i7 priming site, and the i5 priming site is included in the plasmid. The final library was generated using 8 cycles of PCR amplification using NEBNext i5 and i7 dual index primers, and purified cDNA product as template. The resulting product was purified using a DNA Clean and Concentrator-5, and submitted for Illumina high throughput sequencing.

\section{RNA library preparation}

Total RNA was harvested from yeast pellets as described above for qPCR analysis. Extracted RNA from libraries using the pNTI726 or pNTI728 backbones (Fig. 1) was used as the template for reverse transcription by ProtoScript II using sequence-specific primer RM411, according to the manufacturer instructions. Reverse transcription product was purified using a DNA Clean and Concentrator- 5 and used as the template for PCR amplification using i5 and i7 dual indexing primers (NEB \#E7600S).

In CiBER-seq libraries, the barcode is present in the opposite orientation. Reverse transcription was carried out using ProtoScript II according to the manufacturer protocol for oligo-dT priming. Reverse transcription product was treated with $0.5 \mu \mathrm{L}$ RNAse A and $0.5 \mu \mathrm{L}$ RNAse $\mathrm{H}$ for $30 \mathrm{~min}$ to remove RNA, and the DNA product was purified using a DNA Clean and Concentrator-5. The resulting cDNA product was first amplified by PCR for 6 cycles using primers RM511 and RM512 for $P$ (HIS4) and $P$ (PGK1) libraries, or primers RM512 and RM546 for $P(Z)$ libraries. The PCR products were then purified and subsequently amplified in 8 cycles of PCR using NEBNext i5 and i7 dual indexing primers. PCR products were purified using a DNA Clean and Concentrator-5, and submitted for Ilumina highthroughput sequencing.

\section{Barcode to gRNA assignment}

The sequencing library for paired-end barcodeto-gRNA assignment sequencing was constructed by PCR amplification from the barcoded gRNA library using primers RM506 and RM509 for 6 cycles using Q5 polymerase according to the manufacturer instructions. The resulting PCR product was purified using a DNA Clean and Concentrator-5 and then used as a template for a second, 15-cycle PCR using NEBNext i5 and i7 dual indexing primers. The PCR product was purified using a DNA Clean and Concentrator- 5 and analyzed using an Agilent TapeStation 2200 and High Sensitivity D1000 ScreenTape. The PCR product library was then sequenced using $150+150$ base paired-end sequencing on an Illumina MiSeq. Barcode to gRNA assignment sequencing data is available under accession SRR10327353. 


\section{Single gRNA induction}

Yeast strains expressing individual gRNAs were grown overnight in YEPD, then diluted to OD 600 of $0.01 \mathrm{in}$-Leu media containing either $250 \mathrm{ng} /$ $\mathrm{ml}$ anhydrotetracycline, or no inducer. Yeast were harvested 12 hours later (after roughly 6 doublings), pelleted at $4,000 \times g$ for 5 minutes, and pellets were frozen and stored at $-80{ }^{\circ} \mathrm{C}$ for subsequent RNA extraction or protein isolation.

\section{Western blotting}

Total protein was isolated from frozen yeast pellets by resuspending in $1.5 \mathrm{ml}$ of $5 \%$ trichloroacetic acid and incubated at $4^{\circ} \mathrm{C}$ for $<10$ minutes. Protein was pelleted by centrifugation at $16,000 \times g$ for 2 minutes and washed in $0.5 \mathrm{ml}$ acetone, vortexed briefly, and collected by centrifugation for $5 \mathrm{~min}$ at $16,000 \times \mathrm{g}$. Protein pellets were washed once more in $1 . \mathrm{ml}$ acetone, vortexed, and collected by centrifugation for 5 $\min$ at $16,000 \times g$. Pellets were dried overnight and then resuspended in $100 \mu \mathrm{l}$ of freshly prepared protein breakage buffer $(50 \mathrm{mM}$ Tris $\bullet \mathrm{HCl}$ pH 7.5, 1 mM EDTA, 3 mM DTT) containing $100 \mu \mathrm{L}$ of glass beads (BioSpec 11079105). Samples were vortexed in 5 cycles of one minute vortexing followed by one minute on ice. Lysates were transferred to new tubes and collected by centrifugation at $16,000 \times \mathrm{g}$, then resuspended in $150 \mu \mathrm{l}$ resuspension buffer (100 $\mathrm{mM}$ Tris $\bullet \mathrm{HCl} \mathrm{pH} 11,3 \%$ SDS). Samples were boiled for 5 minutes then allowed to cool, and pelleted at $16,000 \times \mathrm{g}$ for 30 seconds. $120 \mu \mathrm{l}$ of lysate was transferred to a new tube, and a BCA assay was performed to determine protein abundance. Equal amounts of total protein were loaded on a $4-12 \%$ polyacrylamide Bis-Tris gel (Thermo Scientific \#NW04120BOX) and separated by electrophoresis in MOPS buffer at $200 \mathrm{~V}$ in a Bolt gel tank (Thermo Scientific \#A25977) according to manufacturer instructions. Protein was then transferred to a nitrocellulose membrane (Thermo Scientific \#88018) according to manufacturer guidelines. Membranes were blocked for 1 hour in TBST with 5\% milk, washed with TBST three times for
10 minutes of shaking, and incubated with primary antibodies in TBST plus $0.5 \%$ milk overnight. Hexokinase loading control was probed with rabbit anti-Hxk2 (Rockland \#100-4159) and phosphorylated eIF2 $\alpha$ was detected using Phospho-eIF2a (Ser52) Polyclonal Antibody (Invitrogen \#44-728G). Membranes were then washed and probed with secondary antibody anti-rabbit IgG, HRP-linked Antibody (Cell Signaling Technology \#7074S). Membrane was imaged by Pierce ECL Western Blotting Substrate (ThermoScientific \#32209) and the chemiluminescence measured on a FluorChem $R$ (ProteinSimple).

\section{Sequencing data analysis}

Sequencing data was processed using Cutadapt to remove constant adapter sequences and demultiplex libraries based on embedded nucleotide identifiers. The adapter sequences for each experimental dataset are provided in Table 6. The underlined sequence represents the library-specific nucleotide identifier.

For dual-guide experiments (Fig. 4), demultiplexing was not required as each pool derived from a single $P$ (HIS4) library. For this reason, adapter trimming was performed by instead taking the first 25 nucleotides using fastx_trimmer.

Trimmed barcodes were then counted using the custom "bc-seqs" program, which collapses barcode variants separated by single-nucleotide mismatches (53). Using the "bc-tabulate" script, these barcode counts were then collected into a matrix, tabulating samples within an experiment. The $P$ (HIS4) and $P(P G K 1)$ libraries were sequenced in two Illumina HiSeq4000 runs and the barcode counts for the runs were summed.

Barcodes were first filtered to remove those that lacked at least 32 counts in the pre-induction DNA sample for at least one replicate. The remaining barcodes were evaluated by differential activity analysis using a massivelyparallel reporter assay linear model (mpralm) 
(60). Barcode expression was compared between samples before and after gRNA induction, or before and after 3AT treatment. Analysis was performed using the aggregate = "sum" parameter to sum barcodes that corresponded to the same guide RNA and model_type = "indep_groups", which treats the replicates as independent experiments. Output tables were merged with gene information from the Saccharomyces Genome Database. Scatterplots comparing log fold change values of individual gRNAs showing were first thresholded for guides in which $q<0.05$ in at least one of the two expression analyses. Barcodes corresponding to no-guide plasmids were used as negative controls to determine the distribution of $p$ values. Genes involved in amino acid biosynthesis, amino-acyl tRNA charging, ISRcontrolled translation initiation, RNA polymerase III transcription, tRNA processing, or actin cytoskeletal arrangement were handcurated and used to annotate volcano and scatter plots. 